\title{
Simulation of acoustic scattering by multiple obstacles in three dimensions
}

\author{
M. Ganesh ${ }^{1} \quad$ S. C. Hawkins ${ }^{2}$
}

(Received 15 August 2008; revised 5 September 2008)

\begin{abstract}
We present an algorithm for simulating acoustic scattering by multiple three dimensional sound-hard, sound-soft, or absorbing impenetrable particles. We demonstrate the high order accuracy of the algorithm by simulating multiple scattering at a range of frequencies. The high order multiple acoustic scattering algorithm in this work facilitates understanding of the interactions of acoustic waves scattered by multiple convex and non-convex bodies.
\end{abstract}

\section{Contents}

1 Introduction

See http://anziamj.austms.org.au/ojs/index.php/ANZIAMJ/article/view/ 1451 for this article, (c) Austral. Mathematical Soc. 2008. Published September 7, 2008. ISSN 1446-8735 
2 Coupled surface integral reformulations

3 Fully discrete algorithms

C36

3.1 Direct approach . . . . . . . . . . . . . . C36

3.2 Boundary decomposition method . . . . . . . . . C38

4 Numerical experiments

C39

References

$\mathrm{C} 43$

\section{Introduction}

Collections of three dimensional impenetrable particles propagate acoustic waves in complex ways, with significant multiple scattering effects between the obstacles, especially when the obstacles are closely spaced. Simulations of multiple acoustic scattering are important for many applications and are computationally challenging in three dimensions. In this work we present an efficient algorithm for time harmonic multiple acoustic scattering by an ensemble $D_{1}, D_{2}, \ldots, D_{J}$ of sound-soft, sound-hard, or absorbing obstacles.

The time harmonic acoustic field $u$ scattered in a homogeneous medium by the impenetrable three dimensional obstacles $D_{1}, D_{2}, \ldots, D_{\text {J }}$ satisfies the Helmholtz equation [3, Page 2]

$$
\Delta u(x)+k^{2} u(x)=0, \quad x \in \mathbb{R}^{3} \backslash \cup_{j=1}^{J} \bar{D}_{j},
$$

where $k=2 \pi / \lambda$ is the wavenumber and $\lambda$ the wavelength, and the Sommerfeld radiation condition (that guarantees the outgoing nature of the waves)

$$
\lim _{|x| \rightarrow \infty}|\boldsymbol{x}|\left(\frac{\partial u}{\partial x}-i k u\right)=0,
$$


where the limit holds uniformly in all directions $\hat{\boldsymbol{x}}=\boldsymbol{x} /|\boldsymbol{x}|$. The scattered field $\mathfrak{u}$, which comprises exterior fields $\mathfrak{u}_{\mathfrak{j}}$ scattered by each obstacle $D_{j}$, with

$$
u=\sum_{j=1}^{J} u_{j},
$$

is induced by an incident field $\mathfrak{u}^{\text {inc }}$ in a way determined by the scattering properties of the surfaces $\partial D_{j}$ of the scatterers $D_{j}$ for $j=1, \ldots, J$.

In the case of scattering by single obstacles (that is, when $J=1$ ), the boundary condition for solving the exterior Helmholtz equation is determined by the way the scattered velocity field (or its normal derivative) interacts with the incident field on the surface of the obstacle. There are three main classes of sound property for impenetrable obstacles, known as sound-soft, sound-hard, or absorbing [3, Page 2], which lead to three distinct boundary condition operators (Dirichlet, Neumann, or Robin/mixed), defined by

$$
\begin{array}{rlrl}
\mathcal{B}^{\text {soft }} v: & =v, \\
\mathcal{B}^{\text {hard }} v:=\frac{\partial v}{\partial \mathfrak{n}}, & \mathcal{B}^{\text {abs }} v:=\frac{\partial v}{\partial \mathfrak{n}}+i \mu v, & \text { (sound-hard/absorbing obstacle) }
\end{array}
$$

where $v$ is a function defined on the surface of the obstacles and $\mathfrak{n}$ is the unit outward normal to the surface. Here $\mu$ is a positive constant parameter determined by the absorbing material properties of the obstacle. In particular, the boundary condition for a single impenetrable obstacle scattering is $\mathcal{B} w=0\left[3\right.$, Page 2], where $\mathcal{B}$ is $\mathcal{B}^{\text {soft }}, \mathcal{B}^{\text {hard }}$ or $\mathcal{B}^{\text {abs }}$ and $\boldsymbol{w}$ is the total field (that is, the sum of the incident and scattered fields).

In the case of multiple obstacle scattering, interactions between the obstacles lead to complicated boundary conditions due to the addition of components scattered by the other obstacles to the field incident on each obstacle. Using the single obstacle scattering boundary conditions, we derive that the 
field $\boldsymbol{u}_{\mathfrak{j}}$ scattered by the $\mathfrak{j}$ th particle satisfies the boundary condition

$$
\mathcal{B} u_{\mathfrak{j}}(\boldsymbol{x})=-\mathcal{B} u^{\mathrm{inc}}(\boldsymbol{x})-\sum_{\substack{j^{\prime}=1 \\ j^{\prime} \neq j}}^{J} \mathcal{B} u_{j^{\prime}}(\boldsymbol{x}), \quad \boldsymbol{x} \in \partial \mathrm{D}_{j}, \quad j=1, \ldots, J .
$$

For multiple scattering the surface normal $\mathfrak{n}$ in the boundary condition operators should be replaced by $\boldsymbol{n}_{\mathfrak{j}}$ on the surface $\partial D_{j}$ for $\boldsymbol{j}=1, \ldots, J$.

The recent book by Martin [6] describes the mathematics of multiple scattering with extensive references to numerical methods for multiple scattering problems. More recent numerical methods for multiple acoustic scattering are in the 2008 paper by Antonie et al. [1] and references therein. These numerical methods and the references cited therein are restricted to scattering by convex sound-soft obstacles. To our knowledge, this is the first work to give an algorithm and simulation results for three dimensional multiple acoustic scattering in configurations that include nearby and well separated convex and non-convex sound-soft, sound-hard, and absorbing deterministic and stochastic particles.

The outline of this paper is as follows. The next section considers the reformulations of the exterior Helmholtz problem as J coupled surface integral equations. Section 3 describes a direct and an iterative fully discrete numerical scheme based on efficient single obstacle acoustic scattering algorithms. We demonstrate these approaches using the single obstacle high order algorithm of Ganesh and Graham [4] for various three dimensional particles with deterministic and stochastic descriptions.

\section{Coupled surface integral reformulations}

For scattering by multiple sound-soft particles, we represent the unique exterior radiating solution $\mathfrak{u}$ of the Helmholtz equation (1), with Dirichlet bound- 
ary condition, as

$u(\boldsymbol{x})=\sum_{j=1}^{J} \int_{\partial \mathrm{D}_{j}}\left[\frac{\partial \Phi}{\partial \mathbf{n}_{\mathfrak{j}}(\mathbf{y})}(\boldsymbol{x}, \mathbf{y})-\mathfrak{i} \gamma \Phi(\boldsymbol{x}, \mathbf{y})\right] v_{j}(\mathbf{y}) \mathrm{ds}(\mathbf{y}), \quad \boldsymbol{x} \in \mathbb{R}^{3} \backslash \cup_{j=1}^{J} \bar{D}_{j}$, where $\Phi(\boldsymbol{x}, \mathbf{y})=e^{i k|x-y|} /(4 \pi|x-y|)$ is a free space Green's function, and the unknown surface densities $v_{j}$ for $j=1, \ldots, J$ are the solution of the system

$$
v_{j^{\prime}}(\boldsymbol{x})+\sum_{j=1}^{J}\left(\mathcal{K}_{j^{\prime} j}-\mathfrak{i} \gamma \mathcal{S}_{j^{\prime} j}\right) v_{j}(\boldsymbol{x})=-2 u^{\mathrm{inc}}(\boldsymbol{x}), \quad \boldsymbol{x} \in \partial \mathrm{D}_{j^{\prime}}, \quad j^{\prime}=1, \ldots, J,
$$

and $\mathcal{K}_{j^{\prime} j}$ and $\mathcal{S}_{j^{\prime} j}$ are the acoustic double and single layer operators corresponding to particles $\boldsymbol{j}$ and $\boldsymbol{j}^{\prime}$, defined for $\boldsymbol{x} \in \partial \mathrm{D}_{\boldsymbol{j}^{\prime}}$ by

$$
\begin{aligned}
\left(\mathcal{K}_{j^{\prime} j} v\right)(\boldsymbol{x}) & =2 \int_{\partial \mathrm{D}_{j}} \frac{\partial \Phi}{\partial \mathbf{n}_{\mathfrak{j}}(\mathbf{y})}(\boldsymbol{x}, \mathbf{y}) v(\mathbf{y}) \mathrm{ds}(\mathbf{y}), \\
\left(\mathcal{S}_{j^{\prime} j} \mathcal{v}\right)(\boldsymbol{x}) & =2 \int_{\partial \mathrm{D}_{j}} \Phi(\boldsymbol{x}, \mathbf{y}) v(\mathbf{y}) \mathrm{ds}(\mathbf{y}) .
\end{aligned}
$$

The unique solution of (5) is guaranteed for all frequencies if $\gamma>0$.

For scattering by an ensemble of absorbing (or sound-hard when $\mu=$ 0 ) scatterers we represent the unique radiating exterior solution $u$ of the Helmholtz equation (1), with mixed or Neumann boundary condition (using $\mathcal{B}^{\text {hard }}=\mathcal{B}^{\text {abs }}$ when $\mu=0$ ) for $\boldsymbol{x} \in \mathbb{R}^{3} \backslash \cup_{j=1}^{J} \bar{D}_{j}$, as [4]

$$
\mathfrak{u}(\boldsymbol{x})=\sum_{j=1}^{\mathrm{J}} \int_{\partial \mathrm{D}_{j}}\left\{\frac{\partial \Phi}{\partial \mathbf{n}_{\mathfrak{j}}(\mathbf{y})}(\boldsymbol{x}, \mathbf{y}) v_{j}(\mathbf{y})+\Phi(\boldsymbol{x}, \mathbf{y})\left[i \mu v_{j}+\mathcal{B}^{\mathrm{abs}} \mathfrak{u}^{\mathrm{inc}}\right](\mathbf{y})\right\} \mathrm{ds}(\mathbf{y})
$$

where the unknown surface densities $v_{j}$ are the solutions of the system

$$
v_{j^{\prime}}(\boldsymbol{x})-\sum_{j=1}^{J}\left(\mathcal{K}_{j^{\prime} j}+i \mu \mathcal{S}_{j^{\prime} j}\right) v_{j^{\prime}}(\boldsymbol{x})=-\left.\sum_{j=1}^{J} \mathcal{S}_{j^{\prime} j} \mathcal{B}^{\text {abs }} u^{\mathrm{inc}}\right|_{\partial D_{j}}(\boldsymbol{x}),
$$


for $\boldsymbol{x} \in \partial \mathrm{D}_{\mathfrak{j}^{\prime}}$ and $\boldsymbol{j}^{\prime}=1, \ldots, \mathrm{J}$. For the unique solvability of (6), we assume that $\mathrm{k}$ is not a (physically meaningless) interior Dirichlet Helmholtz eigenvalue.

\section{$3 \quad$ Fully discrete algorithms}

We write the integral equations (5) and (6) in the general form

$$
v_{j^{\prime}}(\boldsymbol{x})+\sum_{j=1}^{J} \mathcal{M}_{j^{\prime} j} v_{j}(\boldsymbol{x})=h_{j^{\prime}}(\boldsymbol{x}), \quad \boldsymbol{x} \in \partial \mathrm{D}_{j^{\prime}}, \quad j^{\prime}=1, \ldots, J,
$$

where the $h_{j^{\prime}}$ are known functions derived from $u^{\text {inc }}$ via the boundary conditions and each $\mathcal{M}_{j^{\prime} j}$ is one of the weakly singular surface integral operators

$$
\mathcal{M}_{j^{\prime} j}^{\text {soft }}:=\mathcal{K}_{j^{\prime} j}-i \gamma \delta_{j^{\prime} j}, \quad \mathcal{M}_{j^{\prime} j}^{\text {abs }}:=-\mathcal{K}_{j^{\prime} j}-i \mu \delta_{j^{\prime} j} .
$$

We now describe two methods to solve the system of surface integral equations (7). These methods are independent of the discretisation used to solve the integral equations. For each fixed $j^{\prime}=1, \ldots, J$, equation $(7)$ is a single obstacle acoustic scattering problem with a modified right-hand side, and hence we may apply any of the efficient discretisation algorithms to discretise the weakly singular operators after projecting the unknown densities in an appropriate approximation space of, say, dimension $\mathrm{N}$.

\subsection{Direct approach}

The first method that we use for solving (7) is to discretise directly. Discretising (7) leads to the linear system

$$
\mathrm{v}_{j^{\prime}}+\sum_{j=1}^{J} M_{j^{\prime} j} v_{j}=h_{j^{\prime}}, \quad j^{\prime}=1, \ldots, J
$$


where $h_{j^{\prime}}$ for $j^{\prime}=1, \ldots, J$ are known vectors arising from projecting the known source terms $h_{j^{\prime}}$ in (7) onto the approximation space, $M_{j^{\prime} j}$ for $j, j^{\prime}=$ $1, \ldots, J$ are known $N \times N$ matrices obtained by discretising the surface integral terms $\mathcal{M}_{j^{\prime} j} v_{j}$, and for each fixed $j^{\prime}=1, \ldots, J$, the unknown $N$ dimensional vectors $\mathrm{v}_{j^{\prime}}$ (giving linear combination coefficients of the approximate surface densities) must be computed. For simplicity, we assume that a fully discrete Galerkin scheme with an orthonormal basis (and quadrature that integrates the product of any two orthonormal basis functions exactly) has been used, so that discretising the first term in (7) yields the identity matrix in (8). In block matrix form (8) is

$$
\left[\begin{array}{c}
\mathrm{v}^{1} \\
\vdots \\
\mathrm{v}^{\mathrm{J}}
\end{array}\right]+\left[\begin{array}{ccc}
\mathrm{M}_{11} & \ldots & \mathrm{M}_{1 \mathrm{~J}} \\
\vdots & & \vdots \\
\mathrm{M}_{\mathrm{J} 1} & \ldots & \mathrm{M}_{\mathrm{JJ}}
\end{array}\right]\left[\begin{array}{c}
\mathrm{v}^{1} \\
\vdots \\
\mathrm{v}^{\mathrm{J}}
\end{array}\right]=\left[\begin{array}{c}
\mathrm{h}^{1} \\
\vdots \\
\mathrm{h}^{\mathrm{J}}
\end{array}\right] .
$$

The direct approach is applicable for both nearby and well separated obstacles and is efficient in case of orientation changes (or several incident waves), provided that the chosen computing environment allows assembly and storage of the $\mathrm{JN} \times \mathrm{JN}$ matrix. Standard boundary element based algorithms require $\mathrm{N}$ to be of the order hundreds of thousands to millions for low to medium frequency acoustic scattering in three dimensions, see for example details and references given by Ganesh and Graham [4]. Hence for such low order algorithms, the direct approach is prohibitively expensive. However, the high order algorithm by Ganesh and Graham [4] requires only about 2 to $10 \%$ of the unknowns used in standard algorithms and has been demonstrated to be very efficient. Hence the single obstacle scattering algorithm [4] is well suited for the direct approach discretisation of the multiple scattering problem, when the number of particles $J$ is moderate. In some of our computations we avoid the direct approach by using the iterative boundary decomposition technique for well separated obstacles, by solving several $\mathrm{N}$ dimensional systems. However, the boundary decomposition iterates diverge for narrowly separated particles. 


\subsection{Boundary decomposition method}

Using (7), and corresponding to the decomposed boundary condition (4),

$$
\left(I+\mathcal{M}_{j^{\prime} j^{\prime}}\right) v_{j^{\prime}}(\boldsymbol{x})=h_{j^{\prime}}(\boldsymbol{x})-\sum_{\substack{j=1 \\ j \neq j^{\prime}}}^{J} \mathcal{M}_{j^{\prime} j} v_{j}(\boldsymbol{x}), \quad x \in \partial D_{j^{\prime}}, \quad j^{\prime}=1, \ldots, J .
$$

Here each $v_{j^{\prime}}$ is the solution of a single obstacle scattering problem with a modified incident field involving the scattered fields from the other obstacles. These scattered fields are not known but we compute these iteratively and Balabane [2] proved that the sequence $v_{j^{\prime}}=\sum_{m=0}^{\infty} v_{j^{\prime}}^{(m)}$ converges provided the obstacles are sufficiently far apart, where

$$
\left(I+\mathcal{M}_{j^{\prime} j}\right) v_{j^{\prime}}^{(0)}=h_{j^{\prime}}, \quad\left(I+\mathcal{M}_{j^{\prime} j}\right) v_{j^{\prime}}^{(m)}=-\sum_{\substack{j=1 \\ j \neq j^{\prime}}} \mathcal{M}_{j^{\prime} j} v_{j}^{(m-1)}, \quad m=1, \ldots, \infty,
$$

for $j^{\prime}=1, \ldots, J$. The advantage of this iterative method of solution is that only single obstacle scattering problems must be solved, leading to a reduction in complexity and memory requirements. Discretising as in the previous section leads to vectors

$$
v_{j^{\prime}}=\sum_{m=0}^{\infty} v_{j^{\prime}}^{(m)}, \quad\left(I+M_{j^{\prime} j}\right) v_{j^{\prime}}^{(0)}=h_{j^{\prime}}, \quad\left(I+M_{j^{\prime} j}\right) v_{j^{\prime}}^{(m)}=-\sum_{\substack{j=1 \\ j \neq j^{\prime}}}^{J} M_{j^{\prime} j} v_{j}^{(m-1)},
$$

for $j^{\prime}=1, \ldots, J$. The partial sums of (10) are the iterates obtained when solving (9) using the Jacobi method, leading to equivalence with the boundary decomposition method. Physically, $v_{\mathfrak{j}^{\prime}}^{(\mathrm{m})}$ is the field scattered by obstacle $\mathfrak{j}^{\prime}$ induced by incident waves that have undergone $m$ reflections.

To implement the Jacobi method, only the J block matrices on the diagonal, each with dimension only $\mathbf{N} \times \mathbf{N}$, must be inverted. In contrast, 
in the direct approach a $\mathrm{JN} \times \mathrm{JN}$ matrix must be inverted. Because the diagonal blocks are invariant with respect to changes in the location of the obstacles, simulations for many different configurations can be performed with high order algorithms that allow storing the LU factorisations of these blocks, leading to substantially reduced CPU times.

\section{$4 \quad$ Numerical experiments}

In our numerical experiments we discretise using the high order fully discrete single obstacle acoustic scattering algorithm of Ganesh and Graham [4]. The dimension of each block in the block matrix is $N=(n+1)^{2}$ where $n$ is the order of the spectral basis used. As demonstrated in this section for medium frequency multiple scattering by sound-soft, sound-hard, and absorbing particles, it is sufficient that $n \in[60,135]$, depending on the shape and the roughness of the non-convex obstacles with $k a=100$, where $k a$ is the acoustic size with a being the physical diameter of each obstacle in a chosen configuration and $k$ as in (1). With $d$ being the smallest physical distance between the centres of any two particle in the configuration, we say that the configuration has closely located particles, relative to the frequency, if $0<k(d-a) \leq 15$, and well separated particles if $k(d-a)>15$. Since the boundary decomposition approach failed to converge for several closely located configurations, in our simulations we used both the direct and iterative approaches. This highlights the importance of developing high order multiple scattering algorithms, requiring fewer unknowns.

We demonstrate the accuracy of our algorithm by simulating the acoustic cross section (ACS),

$$
\sigma_{n}^{\mathrm{dB}}(\hat{\boldsymbol{\chi}} ; \hat{\mathbf{d}})=10 \log _{10} \sigma_{n}(\hat{\boldsymbol{\chi}}), \quad \sigma_{n}(\hat{\boldsymbol{\chi}} ; \hat{\mathbf{d}})=\lim _{r \rightarrow \infty} 4 \pi r\left|u_{n}(r \hat{\boldsymbol{\chi}})\right|^{2},
$$

computed from the multiple scattered field $u_{n}$. In (11), the unit vectors $\hat{\boldsymbol{\chi}}$ and $\hat{\mathbf{d}}$ are respectively the observation and incident directions, and for 
TABLE 1: Convergence of backscattered ACS $\sigma_{\mathfrak{n}}^{\mathrm{dB}}(-\hat{\mathbf{d}} ; \hat{\mathbf{d}}), \hat{\mathbf{d}}=(-1,0,0)$, for scattering by two sound-soft ice crystals, each with $\mathrm{ka}=100$.

\begin{tabular}{ccccc}
\hline $\mathrm{n}$ & $\mathrm{kd}=109.96$ & $\mathrm{kd}=113.10$ & $\mathrm{kd}=128.81$ & $\mathrm{kd}=131.95$ \\
\hline 105 & 7.410644 & 0.527321 & 2.609607 & 4.725372 \\
115 & 7.408428 & 0.533091 & 2.607145 & 4.728422 \\
125 & 7.407375 & 0.533870 & 2.606579 & 4.728074 \\
135 & 7.407150 & 0.534689 & 2.606542 & 4.728327 \\
\hline
\end{tabular}

TABLE 2: Convergence of backscattered $\operatorname{ACS} \sigma_{n}^{\mathrm{dB}}(-\hat{\mathbf{d}} ; \hat{\mathbf{d}}), \hat{\mathbf{d}}=(0,0,1)$, for scattering by two sound-hard erythrocytes, each with $\mathrm{ka}=100$.

\begin{tabular}{ccccc}
\hline $\mathrm{n}$ & $\mathrm{kd}=103.67$ & $\mathrm{kd}=106.81$ & $\mathrm{kd}=128.81$ & $\mathrm{kd}=131.95$ \\
\hline 60 & 43.81738 & 43.62531 & 43.74709 & 43.64084 \\
65 & 43.81804 & 43.62592 & 43.74764 & 43.64149 \\
70 & 43.81807 & 43.62594 & 43.74766 & 43.64152 \\
75 & 43.81807 & 43.62594 & 43.74766 & 43.64152 \\
\hline
\end{tabular}

monostatic ACS, $\hat{\boldsymbol{x}}=-\hat{\mathbf{d}}$. The ACS is induced by the incident plane wave $u^{i n c}=e^{i k x \cdot \hat{d}}$.

We demonstrate the convergence of our algorithm by tabulating the approximate backscattered ACS $\sigma_{n}^{\mathrm{dB}}(-\hat{\mathbf{d}} ; \hat{\mathbf{d}})$ for pairs of obstacles in broadside configuration with small and large separations. The high order convergence of the multiple scattering algorithm is demonstrated in Tables 1-3 by the increasing number of matching digits in ACS values for small increases in $n$.

The benchmark non-convex multiple obstacles considered in this work are sound-soft, sound-hard, and absorbing beehive shaped obstacles and well known computer models of erythrocytes and random ice crystals. The model erythrocyte (or red blood cells [8]), and hive have concave regions and so are considered difficult obstacles for multiple scattering simulations. In our ice crystal experiments we use several different model ice crystals generated using a stochastic model [7], based on images measured by a cloud particle 

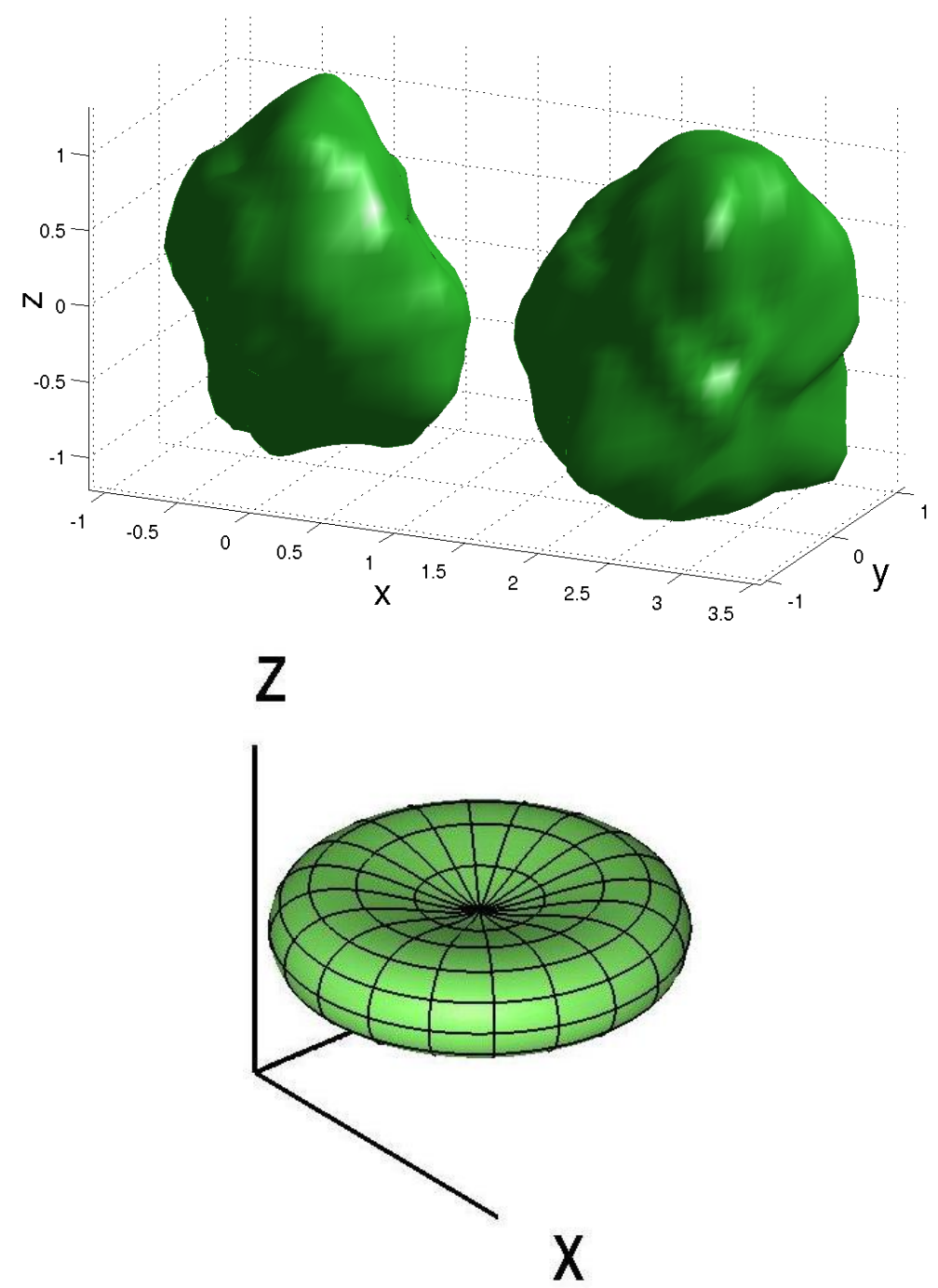

Figure 1: Computer models of stochastic ice crystals and erythrocyte. 
(a)

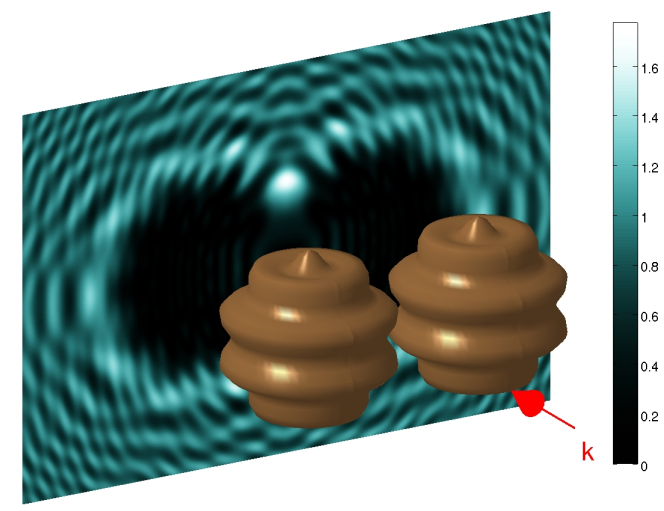

(b)
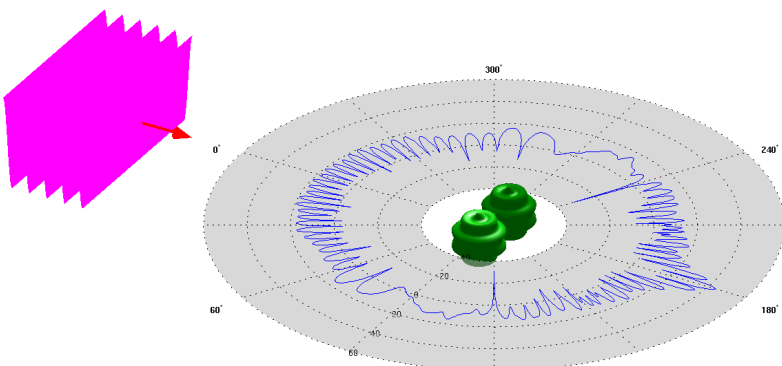

(c)

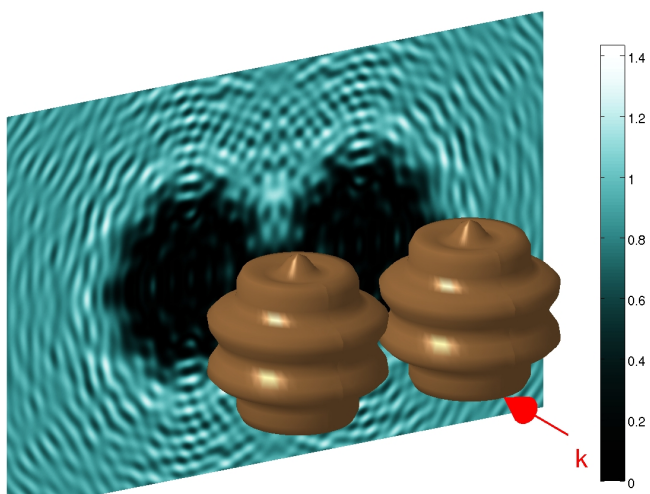

FIGURE 2: Intensity of exterior multiple scattering field $\left(\left|\operatorname{Re}\left(\boldsymbol{u}_{\mathfrak{n}}\right)\right|\right)$ and detection of radiation free (shadow) regions behind two beehive shaped soundsoft obstacles in (a) and absorbing obstacles in (c). Bistatic ACS of two sound-hard obstacles in (b). Results computed with $\mathrm{ka}=100, \mathrm{kd}=103.67$ and $n=125$. 
TABLE 3: Convergence of backscattered ACS $\sigma_{\mathfrak{n}}^{\mathrm{dB}}(-\hat{\mathrm{d}} ; \hat{\mathbf{d}}), \hat{\mathbf{d}}=(-1,0,0)$, for scattering by two absorbing hives, each with $\mathrm{ka}=100$.

\begin{tabular}{ccccc}
\hline $\mathrm{n}$ & $\mathrm{kd}=103.67$ & $\mathrm{kd}=106.81$ & $\mathrm{kd}=128.81$ & $\mathrm{kd}=131.95$ \\
\hline 110 & 18.85959 & 18.20470 & 19.70938 & 17.73657 \\
115 & 18.85955 & 18.20466 & 19.70935 & 17.73652 \\
120 & 18.85956 & 18.20467 & 19.70936 & 17.73653 \\
125 & 18.85957 & 18.20467 & 19.70937 & 17.73654 \\
\hline
\end{tabular}

imager instrument. Some of these models are illustrated in Figure 1 and 2. Further simulation results, obtained using the high order direct and boundary decomposition three dimensional multiple acoustic scattering algorithms, are in a technical report by the authors [5].

Acknowledgments Support of the Australian Research Council and the Colorado Golden Energy Computing Organization (GECO) is gratefully acknowledged. Computations were carried out using the GECO cluster RA.

\section{References}

[1] X. Antoine, C. Chniti, and K. Ramdani. On the numerical approximation of high-frequency acoustic multiple scattering problems by circular cylinders. J. Comput. Phys., 227:1754-1771, 2008. doi:10.1016/j.jcp.2007.09.030 C34

[2] M. Balabane. Boundary decomposition for Helmholtz and Maxwell equations 1: disjoint sub-scatterers. Asymp. Anal., 38:1-10, 2004. http://iospress.metapress.com/content/vu2bd0w9mkem8966 C38

[3] D. Colton and R. Kress. Inverse Acoustic and Electromagnetic Scattering Theory. Springer, 1998. C32, C33 
[4] M. Ganesh and I. G. Graham. A high-order algorithm for obstacle scattering in three dimensions. J. Comput. Phys., 198:211-242, 2004. doi:10.1016/j.jcp.2004.01.007 C34, C35, C37, C39

[5] M. Ganesh and S. C. Hawkins. Simulation of acoustic scattering by multiple obstacles in three dimensions part II: Extended numerical results. Technical report, Colorado School of Mines, 2008. http://www. mines.edu/ mganesh/multiple_08_acoustic_results_part_II.pdf $\mathrm{C} 43$

[6] P. A. Martin. Multiple Scattering: Interaction of Time-Harmonic Waves with $N$ Obstacles. Cambridge University Press, 2006. C34

[7] T. Nousiainen and G. M. McFarquhar. Light scattering by quasi-spherical ice crystals. J. Atmospheric Sci., 61:2229-2248, 2004. doi:10.1175/1520-0469(2004)061;2229:LSBQIC ¿2.0.CO;2 C40

[8] T. Wriedt, J. Hellmers, E. Eremina, and R. Schuh. Light scattering by single erythrocite: Comparison of different methods. J. Quant. Spectrosc. Radiat. Transfer, 100:444-456, 2006. doi:10.1016/j.jqsrt.2005.11.057 C40 


\section{Author addresses}

1. M. Ganesh, Department of Mathematical and Computer Sciences, Colorado School of Mines, Golden, CO 80401, USA. mailto:mganesh@mines . edu

2. S. C. Hawkins, School of Mathematics and Statistics, University of New South Wales, Sydney, NSW 2052, Australia.

mailto:stuart.hawkins@unsw.edu.au 